\title{
émulations
}

\section{Erik Neveu - Sociologie politique des problèmes publics}

\section{Mischa Dekker}

Émulations - Revue de sciences sociales

2018, «Comptes rendus critiques, En ligne »

\section{Article disponible à l'adresse suivante}

https://ojs.uclouvain.be/index.php/emulations/article/view/7413

\section{Pour citer cet article}

Mischa Dekker, «Erik Neveu — Sociologie politique des problèmes publics », Émulations, en ligne. Mise en ligne le 21 avril 2018.

DOI : 10.14428/emulations.cr.041

Distribution électronique : Université catholique de Louvain (Belgique) : ojs.uclouvain.be

(C) Cet article est mis à disposition selon les termes de la Licence Creative Commons Attribution, Pas d'Utilisation Commerciale 4.0 International. http://creativecommons.org/licenses/by-nc/4.0/

Éditeur : Émulations - Revue de sciences sociales / Presses universitaires de Louvain https://ojs.uclouvain.be/index.php/emulations

ISSN électronique : 1784-5734

UCL PRESSES

UNIVERSITAIRES 


\title{
Erik Neveu - Sociologie politique des problèmes publics
}

\begin{abstract}
Mischa Dekker ${ }^{1}$
Recensé : Erik Neveu, Sociologie politique des problèmes publics, Paris, Armand Colin, 2015.

Le 5 octobre 2017, le New York Times publie un premier article où plusieurs femmes accusent le réalisateur Harvey Weinstein de harcèlement et de violence sexuelle. Quelques jours plus tard, le hashtag \#MeToo est lancé. En moins de deux semaines, la question du harcèlement et des violences sexuelles domine les débats dans les journaux aux États-Unis et en Europe. Comment expliquer cette attention croissante pour ce sujet, et comment ce dernier mène-t-il à des changements de politique ou de société ? C'est à de telles questions que la sociologie des problèmes publics essaie de répondre.

Principalement connu pour ses travaux sur les médias, les mouvements sociaux et le genre, le sociologue et politiste de Sciences Po Rennes Érik Neveu nous propose ici un état des recherches en sociologie politique, sur l'étude des " problèmes publics ». Il définit ces derniers comme « les activités d'individus ou de groupe qui produisent des expressions de mécontentement et des revendications relatives à une situation présumée [...] Le problème central pour une théorie des problèmes publics est de rendre compte de l'émergence, de la nature et de la maintenance des activités revendicatives et des réponses qu'elles suscitent » (p. 75-76). Dans Sociologie politique des problèmes publics, l'auteur débute par un constat général : aux États-Unis, à la suite des travaux de Howard Becker (1966), Malcolm Spector et John Kitsuse (1977), et Joseph Gusfield (1963), l'objet des problèmes publics s'inscrit, depuis une vingtaine d'années, dans des entreprises de consolidation d'un champ d'étude à part entière en sociologie. En France, les problèmes publics, comme objet d'étude, se retrouvaient principalement dans des propositions de programmes de recherche et dans des ouvrages collectifs (Cefaï, Terzi, 2012). C'est dans ce contexte que s'inscrit l'ouvrage de Neveu qui propose une formalisation de la sociologie française des problèmes publics.

L'ouvrage se situe dans une tradition "constructiviste» au sens de Ian Hacking (2000) : si un problème public ne s'est pas constitué autour d'une certaine question sociale, ce n'est pas parce qu'il était inévitable qu'il en soit ainsi, ou que le phénomène considéré n'est pas assez grave. Cette posture constructiviste, qui voit dans la construc-
\end{abstract}

${ }^{1}$ Doctorant au Laboratoire interdisciplinaire d'études sur les réflexivités (LIER), Institut Marcel Mauss, EHESS et au laboratoire Political Sociology, Université d'Amsterdam. 
tion d'un problème public une entreprise sociale (Becker, 1966), s'inscrit dans le prolongement des précédents travaux de l'auteur (Neveu, 1999). Ainsi, dans Sociologie politique des problèmes publics, Neveu privilégie l'usage du verbe «cadrer» à celui du terme "cadrage », et souligne la dimension processuelle des problèmes publics. Il met en avant le «principe de symétrie » qui consiste à analyser sur un pied d'égalité tous les acteurs et institutions jouant un rôle dans la construction du problème. Neveu souligne également l'importance de penser la diversité des ressources des acteurs et les effets des propriétés sociales de ces acteurs sur leur positionnement dans le problème public ; des aspects, selon lui, insuffisamment pris en compte par certains sociologues pragmatiques.

C’est ici la multitude des domaines étudiés en sciences sociales qui, d'après l'auteur, complexifie l'entreprise visant à saisir l'objet que constituent les problèmes publics. Néanmoins, « un des plaisirs de ce champ de recherche réside d'ailleurs dans l'extraordinaire diversité des objets et savoirs avec lesquels il met en contact» (p. 15). Les lecteurs sont donc invités à parcourir une multiplicité de débats, auxquels Neveu a luimême contribué ${ }^{2}$ : en sociologie des mouvements sociaux, du travail, de la santé, des médias et de l'action publique. Toutefois, l'auteur construit son ouvrage non pas de façon thématique, mais en le structurant autour de cinq opérations analytiques distinctes : identifier, cadrer, justifier, populariser et mettre en politique. Pour bien restituer ce travail, nous présenterons les différentes étapes analytiques distinguées par Neveu, avec une attention particulière dédiée à son approche constructiviste. La discussion nous amènera à aborder les points suivants : la comparaison, la «mise en politique publique», et l'attention portée aux différends théoriques.

La première opération distinguée par l'auteur pour appréhender un problème public concerne l'«identification » d'un phénomène social comme posant problème. Il constate que les intellectuel.le-s ont un rôle de moins en moins important dans l'agenda des problèmes publics, remplacés par des think-tanks devenus centraux dans la mise en circulation des sujets de discussion. L'auteur distingue ensuite plusieurs types de groupes sociaux actifs dans la mise sur agenda : les mouvements sociaux, les groupes d'intérêt, les partis politiques et les médias.

Dans le chapitre dédié à l'opération relative au fait de "cadrer », Neveu souligne le risque de glisser vers une vision trop intellectualiste de la production des cadrages d'un problème public (Snow et al., 1986). Il s'attaque à une notion purement instrumentaliste des cadres, en insistant sur le fait que le sens de l'action se développe à la fois parallèlement aux cadres qui visent à convaincre le public et en interaction avec eux.

L'auteur présente ensuite une troisième opération, qui est celle de « justifier». Il est pertinent, selon lui, d'isoler analytiquement un travail de justification que l'on peut

${ }^{2}$ On peut notamment citer ses ouvrages Sociologie des mouvements sociaux (1996), Sociologie du journalisme (2001), Introduction aux Cultural Studies (2003, avec Armand Mattelart) et Féminins/Masculins. Sociologie du genre (2004, avec Christine Guionnet). 
associer à une démarche de légitimation des problèmes, de démonstration de leur caractère d'évidence et de gravité. Contrairement au cadrage, la justification engage le bien commun et fait appel à des valeurs partagées.

Le chapitre "populariser», soit la quatrième opération, commence par une exposition de l'agenda-setting theory. L'auteur souligne combien les promoteurs d'informations participent de façon active à la construction d'un problème public, puis met l'accent sur la dimension stratégique de leurs actions. Neveu propose une discussion stimulante et nuancée de l'intérêt heuristique de la notion de « définisseurs primaires », proposée par Stuart Hall (1978) et ses collègues du Center for Contemporary Cultural Studies à Birmingham.

L'auteur présente la dernière opération, la « mise en politique publique », par une discussion des travaux de l'«École de Grenoble», notamment ceux de Bruno Jobert (1994) et de Pierre Muller (1984). La notion de « référentiel d'une politique », c'est-à-dire l'image cognitive qui organise la perception qu'ont les acteurs d'un problème, influe sur la manière dont le problème public se transforme en politique publique. Outre la notion de «référentiel de politique», l'auteur développe un riche inventaire des diverses approches «discursives», notamment les «policy images» de Baumgartner et Jones (2009).

Le dernier chapitre, intitulé « Dire adieu à la culture des problèmes publics?», traite des inclinations récentes, selon l'auteur, qui présentent les problèmes publics de façons individualisée, médicalisée et culturalisée. Ce dernier pose la question de savoir si ces tendances vont à l'encontre d'une culture des problèmes publics, telle que définie par Gusfield (1963).

Revenons à présent sur trois points : la comparaison, la «mise en politique publique », et l'attention portée aux différends théoriques. Premièrement, l'ouvrage, dédié principalement à la situation française, aborde également des faits analysés comme des problèmes publics à l'étranger, notamment aux États-Unis, sans toutefois proposer une discussion de fond relevant des études comparatives sur les problèmes publics. Neveu consacre les deux dernières pages du livre à la question, mais ce fil n'est pas suivi tout au long des chapitres précédents. L'étude comparative de la constitution d'un problème public s'intéressant à plusieurs pays peut par exemple rencontrer la difficulté du traitement d'une même question dans des arènes différentes selon les pays : si elle peut, dans un pays donné, être traitée du point de vue juridique, elle peut l'être, dans un autre, à partir du point de vue éducatif ou médical. Une première étape pour le chercheur en sciences sociales est donc d'illustrer et d'expliquer cette différence de traitement, comme le fait par exemple Abigail Saguy (2003). De ce point de vue, l'ouvrage de Neveu, bien qu'il reconnaisse l'importance des travaux comparatifs, ne fournit que peu de grilles d'analyse et de moyens d'opérationnaliser des études comparatives de la constitution d'un problème public.

Deuxièmement, alors que le chapitre «mise en politique publique» est dédié à la transformation d'un problème en une politique publique, l'hétérogénéité des acteurs 
qui interviennent dans le processus, tel les entreprises, est mentionnée par l'auteur, mais pas abordée. $\mathrm{Si}$, à la lecture de l'ouvrage, la difficulté du choix d'inclure ou d'exclure certains débats est évidente, une discussion sur la manière dont des entreprises privées peuvent s'emparer d'un problème public aurait été enrichissante. Celle-ci aurait pu donner lieu à une démonstration plus précise des conséquences des phénomènes que décrit Neveu, par exemple le New Public Management et le «tournant néolibéral ». Une description plus détaillée de la manière dont un problème se constitue dans des « arènes » spécifiques aurait, ici, permis d'étayer l'analyse proposée dans l'ouvrage. Si Claude Gilbert et Emmanuel Henry (2009) proposent une approche qui prête attention à l'entrée d'un problème public dans une arène spécifique, il aurait été intéressant d'aborder la question de savoir comment un problème entre dans une « arène » plutôt que dans une autre. L'ouvrage aurait aussi pu bénéficier d'un travail théorique plus poussé et d'une définition claire de cette notion-clé de la sociologie des problèmes publics. Quelles stratégies les acteurs utilisent-ils pour rendre une arène plus importante qu'une autre ? Pourquoi, par exemple, choisissent-ils de «médicaliser» ou plutôt de «judiciariser» une question?

Troisièmement, bien que Neveu relève les désaccords théoriques propres à l'étude des problèmes publics, ces derniers sont peu discutés. Pour le premier chapitre, intitulé «identifier », mentionnons, par exemple, les différentes positions théoriques qui peuvent expliquer la mobilisation politique autour d'un thème tel que les concepts de ressources, d'opportunités politiques, mais également d'analyses en termes d'émotion ou d'indignation morale. L'intégration, par exemple, d'éléments plus spécifiques sur les débats entre John McCarthy/Mayer Zald (1977) et David Snow (1986), ou encore entre Doug McAdam (2001) et James Jasper (1997), aurait permis d'éclairer davantage l'opération analytique qu'est le fait de "cadrer », présentée par Neveu dans le deuxième chapitre. De notre point de vue, il aurait été intéressant de montrer comment ces discussions reviennent fréquemment quand sont analysées les diverses opérations identifiées. De la même manière, une présentation plus étoffée des postures respectives de la sociologie critique et de la sociologie pragmatique, quand celles-ci s'intéressent aux problèmes publics, manque parfois à l'ouvrage pour saisir les enjeux qui sous-tendent les débats existant entre ces deux approches.

En dernier lieu, Neveu consacre une partie de l'ouvrage à la mobilisation des recherches scientifiques dans des problèmes publics et discute les travaux sur l'agnotologie, un néologisme introduit par Robert Proctor (2008) pour désigner l'étude de la production culturelle de l'ignorance, des connaissances anti-scientifiques. De ce point de vue, l'ouvrage de Neveu ne fournit que peu de pistes quant aux manières d'articuler l'agnotologie avec le principe méthodologique de symétrie préconisé par l'auteur. Selon ce principe, le ou la sociologue des problèmes publics s'interdit de donner son avis sur le contenu des arguments ou sur la plausibilité des causalités proposées par les acteurs qu'il ou elle étudie. Ce ne serait alors pas de son ressort de décider ce qui est une connaissance anti-scientifique et ce qui au contraire relève de la «vraie science ». À ce 
propos, Neveu souligne la nécessité pour le ou la sociologue de renoncer à faire usage du critère de la "disproportion ", qui invite à distinguer entre des causes qui méritent une plus grande attention et celles qui ne la méritent pas. Une telle prise de position ramènerait le ou la sociologue au statut de claim maker. Mettre en lumière les différends théoriques permet ainsi de déterminer quels sont les outils conceptuels les plus utiles à l'analyse des problèmes publics. Les discussions proposées par l'auteur sur la fin de la culture des problèmes publics nous apparaissent particulièrement pertinentes, si l'on pense, par exemple, à la médiatisation culturalisante, partout en Europe, des agressions sexuelles à Cologne autour du Nouvel An 2016. Quoique son positionnement reste évasif, Neveu semble suggérer qu'afin de bien comprendre ces évolutions, les chercheur-e·s, suivant le principe de symétrie précité, analyseront de la même manière les différentes solutions apportées — sociales, médicales, individualisantes ou culturalisantes - sans préjuger de ces différentes solutions.

La récente campagne \#MeToo montre comment des nouvelles technologies permettent une émergence fulgurante de certains problèmes publics. Cela implique que les chercheur.e-s actifs dans ce champ doivent être continument attentifs à ces innovations. L'un des apports les plus importants de cet ouvrage est l'outillage systématique qu'il fournit pour étudier les problèmes publics de toutes sortes, au moyen des cinq opérations que l'auteur distingue. Si l'on excepte les quelques points que nous venons de soulever, cette Sociologie politique des problèmes publics offre un aperçu stimulant des différents types d'approches utilisés pour enquêter sur les problèmes publics. Une importance particulière est accordée au constructivisme, tout en mettant en lumière la diversité des traditions dans ce champ. Cet ouvrage constitue un excellent aperçu des travaux en sociologie et science politique pour l'ensemble des étudiant-e-s et chercheur.e's qui s'initient à la recherche dans ce champ.

\section{Bibliographie}

BAUMgartner F., JONES B. (2009), Agendas and Instability in American Politics, Chicago, Chicago University Press.

BECKER H. (dir.) (1966), Social Problems. A Modern Approach, New York, Wiley.

Cefaï C., Terzi C. (dir.) (2012), L'expérience des problèmes publics, Paris, Éditions de l'École des hautes études en sciences sociales.

Gilbert C., Henry E. (dir.) (2009), Comment se construisent les problèmes de santé publique, Paris, La Découverte, (« Recherches »).

Gusfield J. (1963), Symbolic Crusades. Status Politics and the American Temperance Movement, Chicago, University of Illinois Press.

HACKING I. (2008 [2000]), Entre science et réalité : la construction sociale de quoi ?, Paris, La Découverte. 
Hall S. (1978), Policing the Crisis: Mugging, the State, and Law and Order, Londres, MacMillan.

HENRY E. (2007), Amiante: un scandale improbable. Sociologie d'un problème public, Rennes, Presses universitaires de Rennes («Res Publica»).

JASPER J. (1997), The Art of Moral Protest, Chicago, University of Chicago Press.

JOBERT B. (dir.) (1994), Le Tournant néo-libéral en Europe, Paris, L’Harmattan.

Mattelart A., Neveu É. (2003), Introduction aux Cultural Studies, Paris, La Découverte.

McAdam D., Tarrow S., Tilly C. (2001), Dynamics of Contention, Cambridge, Cambridge University Press.

MCCARTHY J. D., ZALD M. (1977), «Resource Mobilization and Social Movements: A Partial Theory », American Journal of Sociology, vol. 82, n 6, p. 1212-1241.

MULLER P. (1984), Le technocrate et le paysan, Paris, Éditions ouvrières.

Neveu É. (1996), Sociologie des mouvements sociaux, Paris, La Découverte.

Neveu É. (1999), «L'approche constructiviste des "problèmes publics". Un aperçu des travaux anglo-saxons », Études de communication, vol. 22, p. 41-59.

Neveu É. (2001), Sociologie du journalisme, Paris, La Découverte.

Neveu É., Guionnet C. (2004), Féminins/Masculins. Sociologie du genre, Paris, Amand Colin.

Proctor R., SCHIEBINGer L. (2008), Agnotology: The Making and Unmaking of Ignorance, Redwood City, California, Stanford University Press.

SAGUY A. (2003), What is Sexual Harassment? From Capitol Hill to the Sorbonne, Oakland, University of California Press.

Snow D. A., Rochford B., Worden S. K., Benford R. D. (1986), «Frame Alignment Processes, Micromobilization, and Movement Participation », American Sociological Review, vol. 51, p. 464-481.

SPeCtOR M., Kitsuse J. (1977), Constructing Social Problems, New York, De Gruyter. 